\title{
Generation of Regional Strain Inside Object Using Acoustic Radiation Forces
}

\author{
Yoshifumi Nishio, Naoki Hashimoto, Hideyuki Hasegawa and Hiroshi Kanai \\ Graduate School of Engineering, Tohoku University \\ Sendai 980-8579, Japan \\ Email: hkanai@ecei.tohoku.ac.jp
}

\begin{abstract}
Recently, there are some reports on assessment of the mechanical properties of tissue by measuring its minute displacement induced by acoustic radiation force which is generated by two ultrasounds at slightly different frequencies. However, when an object exists in the soft tissue, these methods may generate only the change in position of the object, and the strain of the object is hardly generated. Therefore, in such cases, the mechanical properties of the object can not be measured. Even in such cases, we aim to generate the regional strain inside the object by using two phase-controlled acoustic radiation forces. We realize the generation of the strain inside the object by setting the phase difference between two cyclic acoustic radiation forces, which are radiated at two different depth points along the ultrasonic beam in the object, to be 180 degrees. The intensity of the applied continuous ultrasonic wave is suppressed to be lower than the safety guideline $\left(1 \mathrm{~W} / \mathrm{cm}^{2}\right)$ recommended by the Japan Society of Ultrasonics in Medicine (JSUM). A minute strain with amplitude of less than $1 \mu \mathrm{m}$ is cyclically generated by the radiation force with a low frequency $\Delta f$ of several Hertz. For simultaneous measurement of the minute strain with ultrasound, cyclic radiation force is applied intermittently by maintaining its envelope waveform of the low-frequency component of $\Delta f \mathbf{H z}$. At the same time, an ultrasonic correlation-based method, namely, the ultrasonic phased tracking method, is employed to measure the minute strain. In basic experiments, the minute strain of several micrometers was generated in a gel phantom by applying radiation forces, and was successfully measured by the ultrasonic phased tracking method.
\end{abstract}

\section{INTRODUCTION}

In recent years, some remote actuation methods based on acoustic radiation force have been reported. Fatemi and coworkers proposed an imaging method called ultrasoundstimulated acoustic emission (USAE) [1], [2]. Their system consists of two confocal ultrasonic transducers, and two ultrasound beams with two slightly different frequencies of $f$ and $(f+\Delta f)$ are transmitted. Acoustic radiation pressure, $P_{\mathrm{R}}(t)$, exerted on the interface between two different media is a function of the energy density, $e(t)$, and the specific acoustic impedances, $Z_{1}$ and $Z_{2}$, of the media [3]. The energy density, $e(t)$, is proportional to the square of the sum of the sound pressures, $p_{1}(t)$ and $p_{2}(t)$, generated by the two transducers. In the intersectional area of the two beams, therefore, an oscillatory radiation pressure $P_{\mathrm{R}}(t)$ with the frequency difference, $\Delta f$, is applied to the interface. The radiation force produces acoustic emission which is closely related to the mechanical frequency response of the medium. By measuring the acoustic emission with a hydrophone, hard inclusions in a soft material were experimentally detected. The spatial resolution in the depth direction corresponds to the size of the intersectional area.

Nightingale et al. proposed an alternative imaging method in which the pulsed ultrasound is employed for applying the radiation force to a soft tissue during short durations (less than $1 \mathrm{~ms}$ ). The viscoelastic properties of the tissue are measured from the magnitude and the transient response of the displacement, $d(t)$, of the tissue [4]. In order to generate measurable displacement, $d(t)$, by several ultrasonic pulses, high-intensity pulsed ultrasound of $1,000 \mathrm{~W} / \mathrm{cm}^{2}$ is employed. According to the safety guideline shown by JSUM, however, the intensity of ultrasound is recommended to be less than 240 $\mathrm{mW} / \mathrm{cm}^{2}$ and $1 \mathrm{~W} / \mathrm{cm}^{2}$, for the pulsed wave and the continuous wave, respectively [5]. Therefore, the intensity of the pulsed ultrasound employed by Nightingale et al. is far greater than that indicated in the safety guideline.

To improve the spatial resolution, Michishita et al. used ultrasound correlation-based method, the ultrasonic phased tracking method [6], to measure the minute displacement, $d(t)$, caused by acoustic radiation force. Moreover, to suppress the sound pressure of the employed ultrasound, two continuouswave (CW) ultrasound beams of $f$ and $f+\Delta f$ are employed to cyclically actuate the acoustic radiation pressure, $P_{\mathrm{R}}(t)$, at low frequency $\Delta f$ to increase the signal-to-noise ratio in displacement estimation [7].

However, as illustrated in Fig. 1(a), when the elastic modulus of the media is far smaller than that of the object, an acoustic radiation force may generate only the change in position of the object. In such cases, the mechanical properties of the object cannot be estimated. In this paper, to generate the strain of the object even in such cases, two acoustic radiation forces with phases of $\theta$ and $\theta+\pi$ were applied at two different positions in the object as shown in Fig. 1(b). Furthermore, the displacements of two points, where the two radiation forces were applied, were measured by the ultrasonic phased tracking method.

\section{PRINCIPLE}

As illustrated in Fig. 2, when a continuous plane-wave ultrasound is incident on an interface between two different media, a constant force, which is called an acoustic radiation force, is exerted on the interface. Acoustic radiation pressure, $P_{\mathrm{R}}(t)$, is defined as the acoustic radiation force per unit area 


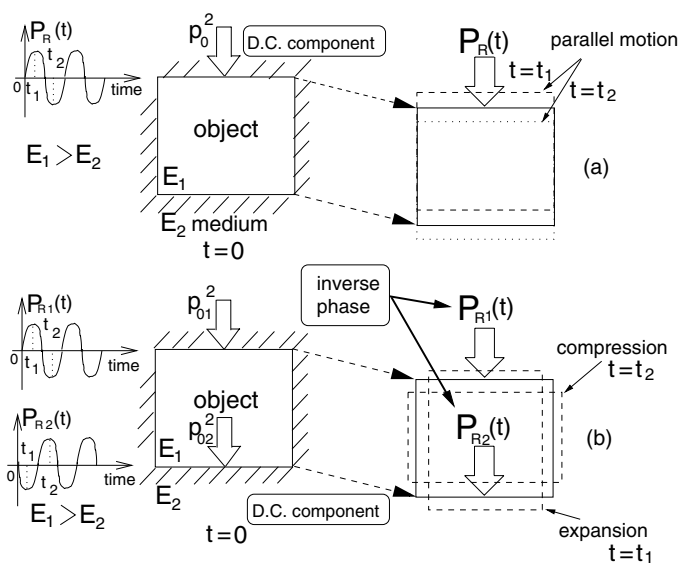

Fig. 1. (a) A model of a parallel motion of an object using one acoustic radiation force. (b) A model of a strain of an object using two acoustic radiation forces.

of the interface as follows:

$$
P_{\mathrm{R}}(t)=\left(1+R^{2}\right) e(t),
$$

where $R$ and $e(t)$ are the reflection coefficient of sound pressure and the energy density at the interface, respectively. In eq. (1), the transmitted wave is assumed to be perfectly absorbed in the object. Using the densities, $\rho_{1}$ and $\rho_{2}$, and sound speeds, $c_{1}$ and $c_{2}$, of the media and object, the reflection coefficient, $R$, and the energy density, $e(t)$, are defined by

$$
\begin{aligned}
R & =\frac{Z_{2}-Z_{1}}{Z_{2}+Z_{1}} \\
& =\frac{\rho_{2} c_{2}-\rho_{1} c_{1}}{\rho_{2} c_{2}+\rho_{1} c_{1}}, \\
e(t) & =\frac{1}{\rho_{1} c_{1}^{2}}\{p(t)\}^{2},
\end{aligned}
$$

where $p(t)$ is the sound pressure at the interface. JSUM shows the safety guideline, in which the intensity of the CW ultrasound should be less than $1 \mathrm{~W} / \mathrm{cm}^{2}$. By assuming that density $\rho_{1}$ and sound speed $c_{1}$ of water are $10^{3} \mathrm{~kg} / \mathrm{m}^{3}$ and $1,500 \mathrm{~m} / \mathrm{s}$, respectively, the acoustic radiation pressure, $P_{\mathrm{R}}(t)$, exerted on the interface of the totally absorbing object $(R=0)$ is calculated as $6.67 \mathrm{~Pa}$ when the ultrasound intensity is 1 $\mathrm{W} / \mathrm{cm}^{2}$.

The energy density, $e(t)$, of the incident wave is proportional to the square of the sound pressure, $p(t)$, of the ultrasound beam. When two ultrasonic beams with slightly different frequencies, $f$ and $f+\Delta f$, are crossed each other, the sound pressure, $p_{\text {sum }}(t)$, at the intersectional area is expressed by the sum of sound pressures of two ultrasonic beams as follows:

$$
p_{\text {sum }}(t)=p_{0} \cos \omega t+p_{0} \cos (\omega+\Delta \omega) t,
$$

where $p_{0}, \omega$, and $\Delta \omega$, are the amplitude of the sound pressure of each ultrasound beam, angular frequency of the incident wave $(\omega=2 \pi f)$, and difference in angular frequency $(\Delta \omega=$

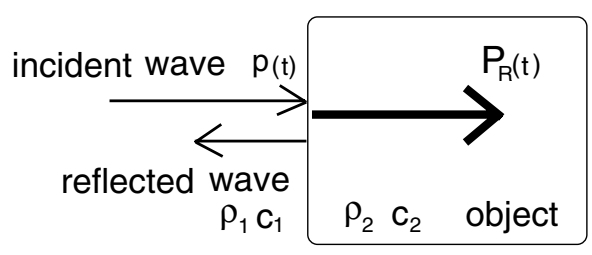

Fig. 2. A model of the acoustic radiation force exerted on the interface of the object.

$2 \pi \Delta f)$, respectively. For this case, the energy density, $e(t)$, is given by

$$
\begin{aligned}
e(t)= & \frac{1}{\rho_{1} c_{1}^{2}}\left\{p_{\text {sum }}(t)\right\}^{2} \\
= & \frac{1}{\rho_{1} c_{1}^{2}}\left\{p_{0} \cos \omega t+p_{0} \cos (\omega+\Delta \omega) t\right\}^{2} \\
= & \frac{p_{0}^{2}}{\rho_{1} c_{1}^{2}}\{1+\cos \Delta \omega t+\cos (2 \omega+\Delta \omega) t \\
& \left.\quad+\frac{1}{2} \cos 2 \omega t+\frac{1}{2} \cos 2(\omega+\Delta \omega) t\right\} .
\end{aligned}
$$

From the second term of the right-hand side of eq. (5), it is found that the energy density, $e(t)$, of the incident field has a component with the frequency difference $\Delta f$. Therefore, the cyclically oscillating radiation pressure, $P_{\mathrm{R}}(t)$, at the frequency difference $\Delta f$ is given by

$$
P_{\mathrm{R}}(t)=\left(1+R^{2}\right) \frac{p_{0}^{2}}{\rho_{1} c_{1}^{2}} \cos \Delta \omega t .
$$

Thus, using an ultrasound beam generated by the sum of signals at the slightly different frequencies, $f$ and $f+\Delta f$, the oscillatory radiation force can be cyclically applied to the focal area of the beam.

In this paper, in order to generate the regional strain inside the object, we use two phase-controlled acoustic radiation pressures, $P_{\mathrm{R} 1}(t)$ and $P_{\mathrm{R} 2}(t)$, given by

$$
\begin{gathered}
P_{\mathrm{R} 1}(t)=\left(1+R^{2}\right) \frac{p_{01}^{2}}{\rho_{1} c_{1}^{2}} \cos \Delta \omega t, \\
P_{\mathrm{R} 2}(t)=\left(1+R^{2}\right) \frac{p_{02}^{2}}{\rho_{1} c_{1}^{2}} \cos (\Delta \omega t+\pi) .
\end{gathered}
$$

As shown in Fig. 1(b), by applying these phase-controlled acoustic radiation pressures at two points of the object, the regional strain is generated inside the object.

\section{EXPERIMENTAL SETUP}

An experimental setup is illustrated in Fig. 3. In order to measure the strain, we employed ultrasonic diagnostic equipment (Toshiba SSH-160A) with a sector-type probe (center frequency: $5 \mathrm{MHz}$ ). The equipment was modified to detect the minute strain of the object by the ultrasonic phased tracking method. An object made of gel (diameter: $40 \mathrm{~mm}$, height: $20 \mathrm{~mm}$, containing carbon to obtain a strong reflection wave) was placed at the bottom of a water tank. For the application of radiation pressures, $P_{R 1}(t)$ and $P_{R 2}(t)$, two concave 


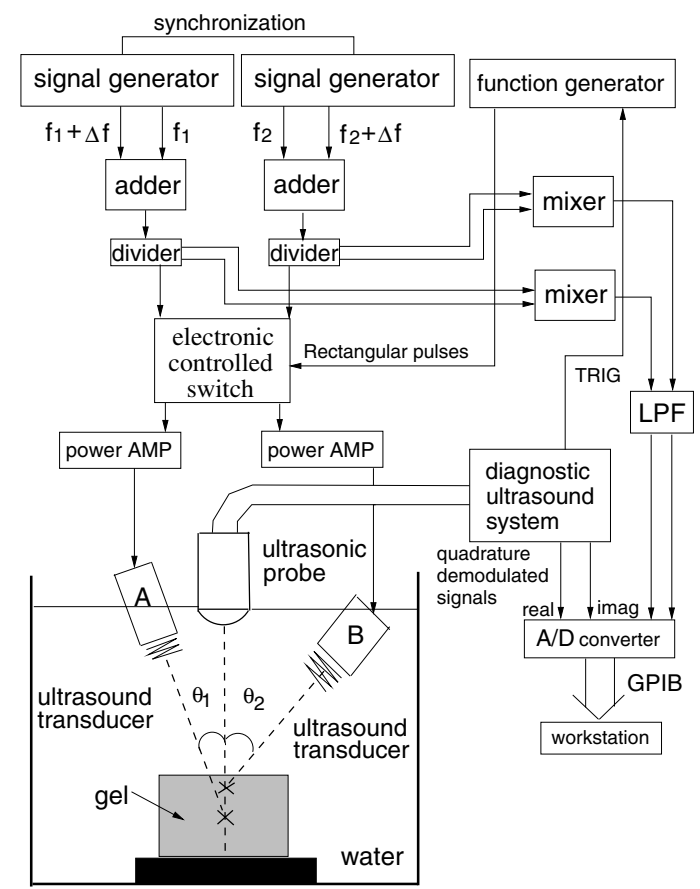

Fig. 3. Experiment setup for measurement of the strain inside the object cyclically actuated using two concave ultrasonic transducers.

ultrasonic transducers were employed. A concave ultrasonic transducer (Tokimec 1Z20I-PF50-C; center frequency: $1 \mathrm{MHz}$ ) was driven by a sum of two continuous waves (CWs) at two slightly different frequencies of $1 \mathrm{MHz}$ and $1 \mathrm{MHz}+\Delta f \mathrm{~Hz}$. The resultant ultrasound beam was focused at $45 \mathrm{~mm}$ below the surface of the transducer, and the focal point was set at 5 $\mathrm{mm}$ deeper than the top of the object with a beam angle, $\theta_{1}$, from the vertical axis of 22 degrees. Another concave transducer (Tokimec 3Z13I-C-PF50-K353; center frequency: $3 \mathrm{MHz}$ ) was driven by a sum of CWs at $3 \mathrm{MHz}$ and $3 \mathrm{MHz}+$ $\Delta f \mathrm{~Hz}$ with a phase difference of 180 degrees in comparison with the sinusoidal wave $\cos \Delta \omega t$ of the first actuation. The focal point, which is $45 \mathrm{~mm}$ away from the surface of the transducer, was set at the top of the object with a beam angle, $\theta_{2}$, of 40 degrees.

In this system, the CW ultrasound for actuation will interfere in the pulsed ultrasound for strain measurement. In order to avoid this interference, it is necessary to stop actuation during transmission and reception of the ultrasonic pulse for the measurement. Therefore, we used an electrical switch to control the cessation of the CW ultrasound for actuation [7].

\section{EXPERIMENTAL RESUlts}

Figs. 4(a) and 5(a) show the M-mode images of the object. In each figure, the difference frequency $\Delta f=10 \mathrm{~Hz}$ was employed. Acoustic radiation pressures, $P_{\mathrm{R} 1}(t)$ and $P_{\mathrm{R} 2}(t)$, shown in Figs. 4(b) and 5(b) were calculated based on eq. (6) as follows: The density, $\rho_{2}$, and the sound speed, $c_{2}$, of the object were measured as $1.1 \times 10^{3} \mathrm{~kg} / \mathrm{m}^{3}$ and $1.47 \times 10^{3}$ $\mathrm{m} / \mathrm{s}$, respectively, in a separate experiment. By assuming the

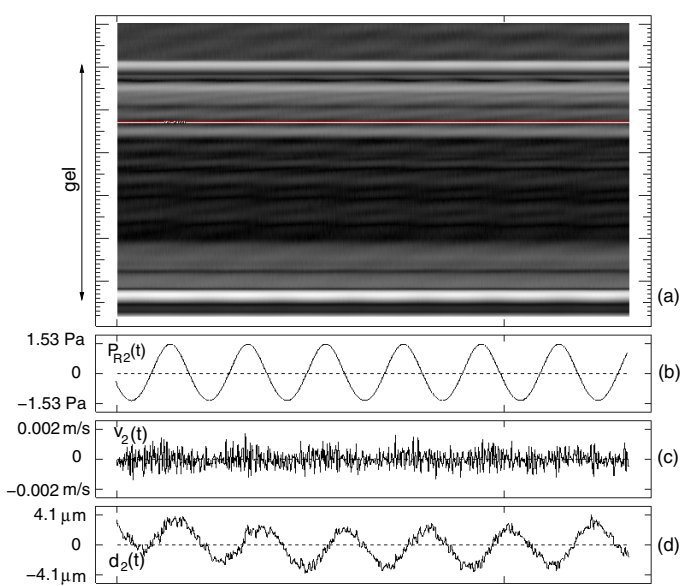

Fig. 4. The results of this experiment radiating at $3 \mathrm{MHz}$. (a) M-mode image of gel. (b) The estimated acoustic radiation pressure, $P_{\mathrm{R} 2}(t)$, given by 6 . (c) The vibration velocity of the object, $v_{2}(t)$, obtained by the ultrasound phased tracking method, (d) The displacement of the object, $d(t)$.

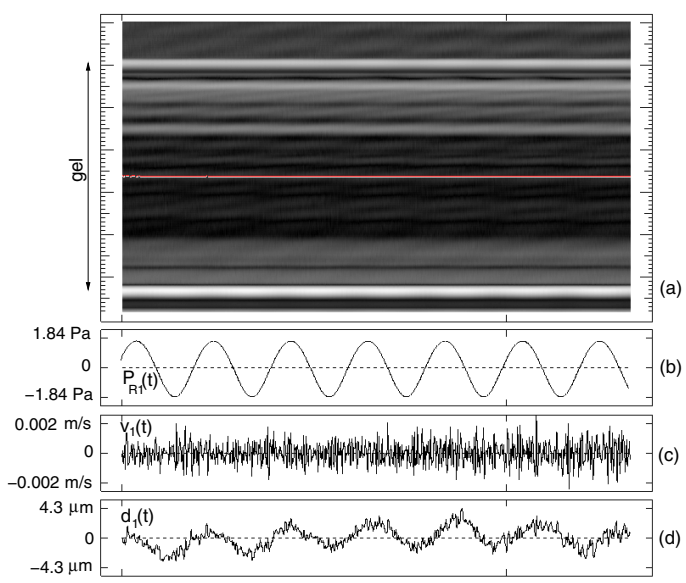

Fig. 5. The results of this experiment radiating at $1 \mathrm{MHz}$. (a) M-mode image of gel. (b) The estimated acoustic radiation pressure, $P_{\mathrm{R} 1}(t)$, given by 6 . (c) The vibration velocity of the object, $v_{1}(t)$, obtained by the ultrasound phased tracking method, (d) The displacement of the object, $d(t)$.

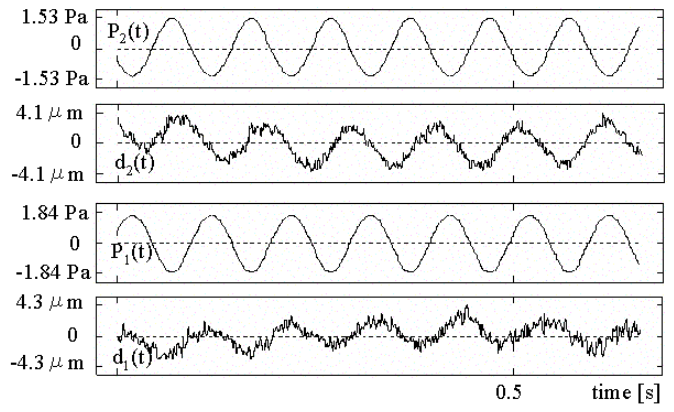

Fig. 6. comparing displacement actuated by two acoustic radiation forces at two positions. 
density, $\rho_{1}$, and the sound speed, $c_{1}$, of water to be $1.0 \times 10^{3}$ $\mathrm{kg} / \mathrm{m}^{3}$ and $1.5 \times 10^{3} \mathrm{~m} / \mathrm{s}$, respectively, the pressure reflection coefficient, $R$, and the energy reflection coefficient, $R^{2}$, were respectively calculated as 0.038 and 0.0014 using eq. (2). By measuring the acoustic fields of the each ultrasound for actuation with a hydrophone (Force Institute, MH-28-10), we estimated the amplitude of the focused sound pressures of $p_{01}$ for $1-\mathrm{MHz}$ transducer and $p_{02}$ for $3-\mathrm{MHz}$ transducer. The amplitudes of the focused sound pressure, $p_{01}$ and $p_{02}$, were measured beforehand and plotted as a function of the voltage applied to the transducers, as shown in Figs. 7 and 8. In this study, the amplitudes of the applied voltages, $\mathrm{V}_{0}$, were set to be $90 \mathrm{~V}$ for $1 \mathrm{MHz}$ transducer and $23 \mathrm{~V}$ for $3 \mathrm{MHz}$ transducer, to make the amplitudes of the acoustic radiation forces almost similar. By neglecting the attenuation of the media surrounding the object, the amplitudes of the sound pressures, $p_{01}$ and $p_{02}$, were calculated to be $130 \mathrm{kPa}$, respectively.

In this paper, by assuming the object to be a totally absorbing material $(R=0)$ and considering the angles, $\theta_{1}$ and $\theta_{2}$, of the transducer for the actuation from the vertical axis, the vertical components of the acoustic radiation pressures, $P_{\mathrm{R} 1}(t)$ and $P_{\mathrm{R} 2}(t)$, exerted on the two points in the object were calculated as $1.84 \mathrm{~Pa}$ for $1 \mathrm{MHz}$ transducer and $1.53 \mathrm{~Pa}$ for $3 \mathrm{MHz}$ transducer.

The vibration velocities, $v_{1}(t)$ and $v_{2}(t)$, at two different points in the object actuated by the acoustic radiation forces were measured by the ultrasonic phased tracking method, as shown in Figs. 4(c) and 5(c). By integrating the velocities, $v_{1}(t)$ and $v_{2}(t)$, the displacements of these two points, $d_{1}(t)$ and $d_{2}(t)$, were obtained as shown in Figs. 4(d) and 5(d), respectively. In Figs. 4(d) and 5(d), it is found that the object was cyclically actuated at the frequency difference, $\Delta f$, of 10 $\mathrm{Hz}$ with an amplitude of the order of a few micrometers [7]. The difference in the phase of the acoustic radiation forces is 180 degrees. However, the difference in the phase of the measured displacements at two points is 120 degrees. The amplitudes of the measured displacements of these two points were $3.5 \mu \mathrm{m}$ actuated by $1 \mathrm{MHz}$ transducer and $4.8 \mu \mathrm{m}$ actuated by $3 \mathrm{MHz}$ transducer. From these results, using the proposed method, the regional strain inside object was successfully generated and measured with ultrasound.

\section{CONCLUSION}

In this study, in order to generate the regional strain inside the object, we constructed an experimental setup for application of cyclic remote actuation in the object using two focused ultrasonic transducers. The phase difference between two acoustic radiation forces, which were respectively applied at two different positions in the object, was controlled to be 180 degrees. The resulting displacements of these two positions were successfully measured by the ultrasonic phased tracking method. The phase difference of the measured displacements was 120 degrees. These results show potential of the proposed method for generation of the regional strain inside the object for tissue characterization.

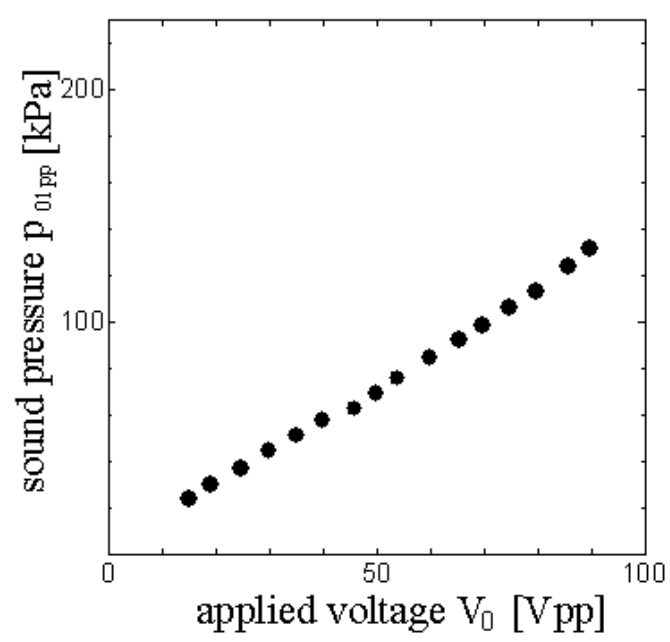

Fig. 7. Sound pressure $p_{01}$ at the focused point plotted as a function of applied voltage $\mathrm{V}_{0}$ for $1 \mathrm{MHz}$ transduer.

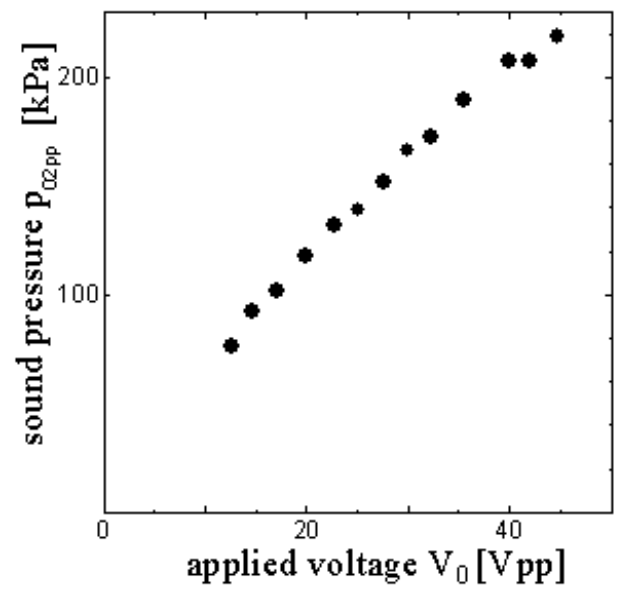

Fig. 8. Sound pressure $p_{02}$ at the focused point plotted as a function of applied voltage $\mathrm{V}_{0}$ for $3 \mathrm{MHz}$ transduer.

\section{REFERENCES}

[1] M. Fatemi, and J. F. Greenleaf, "Vibro-Acoustic Mammography," IEEE Transactions on Medical Imaging, Vol. 21, pp. 1-8, 2002.

[2] M. Fatemi, and J. F. Greenleaf, "Vibro-acoustgraphy: An imaging modality based on ultrasound-stimulated acoustic emission," Proceedings of the National Academy of Sciences of the United States of America, Vol. 96, pp. 6603-6608, 1999.

[3] G. R. Torr, "The acoustic radiation force," American Journal of Physics, Vol. 52, pp. 402-408, 1984.

[4] K. Nightingale, M. S. Soo, R. Nightingale, and G. Trahey, "Acoutic radiation force impulse imaging: in vivo demonstration of clinical feasibility," Ultrasound in Medicine and Biology, Vol. 28, pp. 227-235, 2002.

[5] JSUM: Jpn. J. Med. Ultrason. Vol. 11 (1984) 41 [in Japanese].

[6] H. Kanai, M. Sato, Y Koiwa, and N. Chubachi, "Transcutaneous Measurement and Spectrum Analysis of Heart Wall Vibrations," IEEE Transactions on Ultrasonics, Fellowelectrics, and Frequency Control, Vol. 43, pp. 791-810, 1996.

[7] K. Michishita, H. Hasegawa and H. Kanai, "Ultrasonic Measurement of Minute Displacement of Object Cyclically Actuated by Acoustic Radiation Force," Japanese Journal of Applied Physcis Vol. 42 pp. 4608$4612,2003$. 